\title{
3D Imaging by Laser Scanning Confocal Microscopy
}

\author{
Liang Liang*, Kirk Czymmek** \\ * DuPont Central Research and Development, P. O. Box 80228, Wilmington, DE 19880 USA \\ **15 Innovation Way, Delaware Biotechnology Institute, University of Delaware, Newark, DE \\ 19711 USA
}

One of the greatest advances in light microscopy within the past two decades is the development of laser scanning confocal microscopy (LSCM) [1]. By use of a pinhole to exclude the collection of light from out-of-focus regions, LSCM provides a non-destructive method for acquiring high contrast, three-dimensional data with improved resolution over its conventional light microscopy counterpart (See Fig. 1 for the principle of LSCM). This is achieved by raster scanning a laser in the $\mathrm{x}-\mathrm{y}$ plane and the user defined movement of the microscope objective lens in the $\mathrm{z}$ direction to produce a series of "optical" sections. Recently, multiphoton microscopy which utilizes nearinfrared radiation (NIR) to generate images without the use of a pinhole enables improved depth of penetration for thick specimens, restricts photobleaching and phototoxic effects to the focal plane and is potentially less damaging to living specimen when compared to visible and UV light [2]. Regardless of the optical sectioning approach, samples are routinely imaged as deep as several hundreds microns from their surface and within several minutes full three-dimensional (3-D) volumes of data are generated. Coupled with powerful techniques such as fluorescence recovery after photobleaching (FRAP), fluorescence resonance energy transfer, four-dimensional data acquisition, spectral imaging and high-end volumetric data analysis software tools, confocal and multiphoton microscopy have proven to be essential research equipment in many fields.

An increasing number of biological and material science applications have been developed using LCSM in recent years [3]. Optically transparent organic and inorganic materials are well suited for LSCM microstructure characterization (Figs. 2 \& 3). Non-transparent materials may be imaged in reflection mode to create 3-D surface renditions without coating or other destructive preparative treatments. In addition, 3-D images of multi-phased materials have been readily obtained with little sample preparation time. For biological samples, a vast array of fluorescent antibodies and probes may be applied to derive specific chemical information (Fig. 4). A rainbow of florescent proteins also can be expressed in genetically amenable organisms allowing visualization of a multitude of targets in living cells without the addition of exogenous markers.

Like many microstructural characterization techniques, LSCM has its own limitations. Axial and lateral resolution are low when compared to electron microscopy. In addition, opaque materials, poorly labeled or unlabeled samples, and light-scattering materials may prove problematic for imaging to name just a few. Thus, it is advisable to use multiple complementary microscopy techniques to verify and solve real-life academic research and industrial applications.

References:

[1] J. Pawley, Editor, Handbook of Biological Confocal Microscopy, $2^{\text {nd }}$ Ed., Plenum Press, New York (1995)

[2] W. Denk et al., Science 248 (1990) 73

[3] A. E. Ribbe, Trends in Polymer Science 5 (1997) 333 

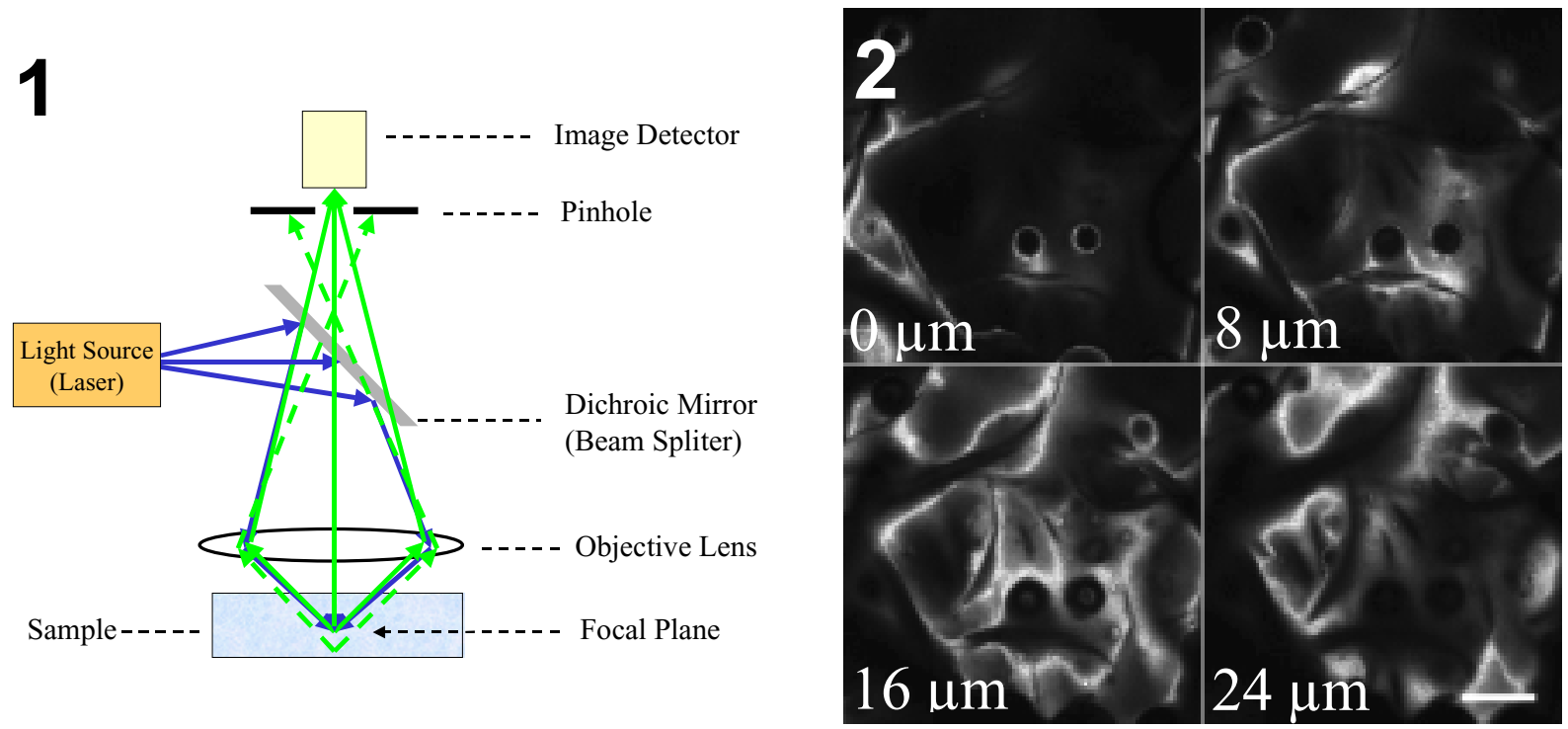

Fig. 1. Schematic representation of LSCM principle. Laser light is raster-scanned across the sample point-by-point using a confocal pinhole in front of the image detector to reject out-offocus light from the final image. Both confocal and multiphoton microscopy are able to generate optical sections and with user-defined movement of the objective focal plane, 3-D z-stacks of data can be obtained. Fig. 2. A gallery of 4 optical sections at $8 \mu \mathrm{m}$ intervals selected from an original z-stack of 80 images acquired with a $1 \mu \mathrm{m}$ z-interval. Bar $=75 \mu \mathrm{m}$.
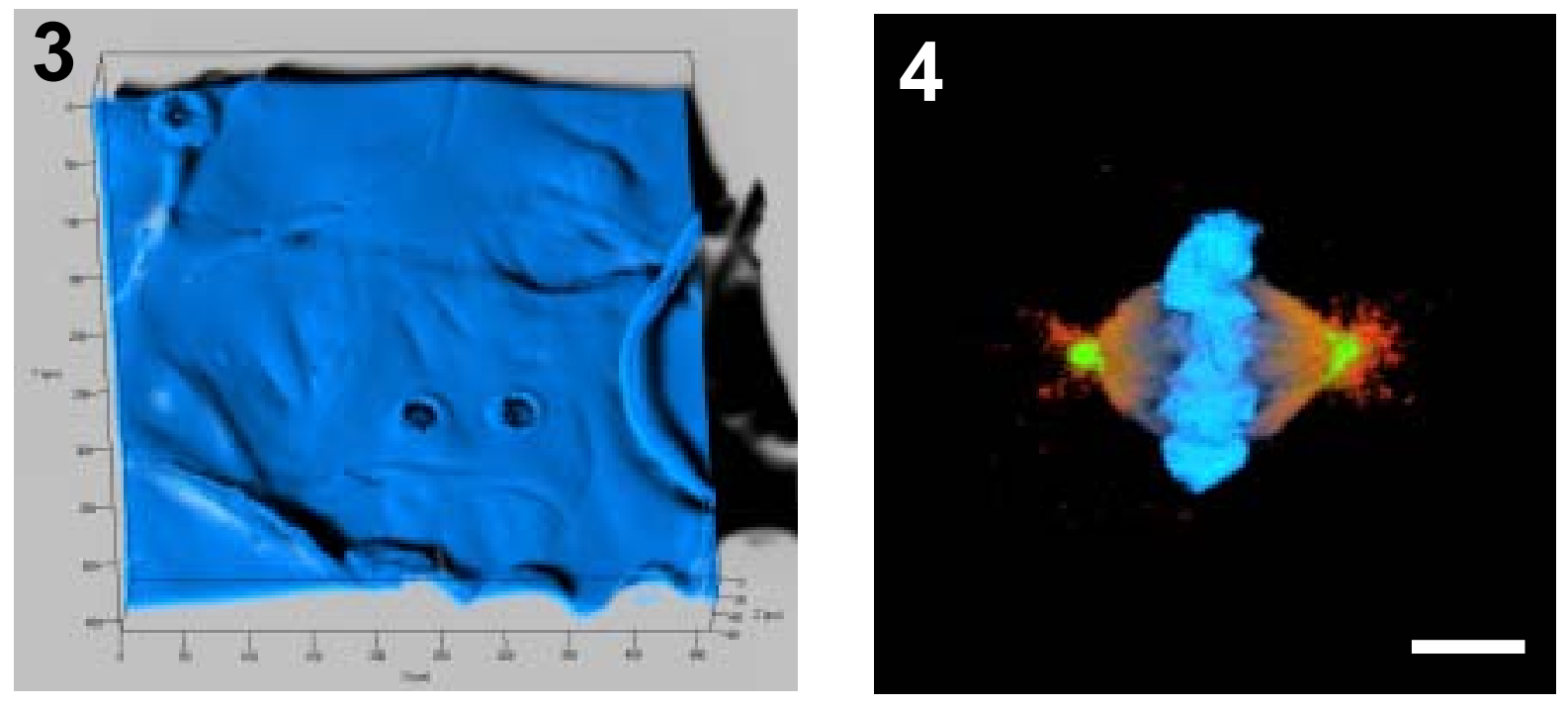

Figs. 3 and 4. Multiphoton/confocal 3-D renderings. Fig. 3. A 3-D shadow projection image of the same z-stack as Fig. 2 gallery above illustrating surface features of fluorescent lettering imprinted on cloth. Fig. 4. 3-D shadow projection of a mitotic cell with antibody labeled centrioles (green), spindle microtubules (orange-red) and Hoecsht 33258 counterstained chromosomes (blue). Bar $=3 \mu \mathrm{m}$. Both 3-D images created with Carl Zeiss, VisArt software. 